\title{
A computationally efficient hybrid leakage model for positive displacement compressors and expanders
}

\author{
Ian H. Bell ${ }^{a, *}$, Eckhard A. Groll ${ }^{b}$, James E. Braun ${ }^{b}$, W. Travis Horton ${ }^{b}$ \\ ${ }^{a}$ University of Liège, Department of Aerospace and Mechanical Engineering, Liège, Belgium \\ ${ }^{b}$ Purdue University Department of Mechanical Engineering, 140 S. Martin Jischke Drive, West Lafayette, IN, 47906
}

\begin{abstract}
An empirical frictional correction factor to the isentropic nozzle model has been developed for application to refrigerant leakage modeling in scroll, rotary and other similar compressors. This correction factor is derived by calculating the leakage mass flow rate with a compressible, variable area, real gas properties model and referencing the results to an isentropic nozzle model. The ratio of flows is correlated to the Reynolds number, a characteristic length and the leakage gap width. A representative selection of fluids and geometries are employed. For all the correlations, at least $93 \%$ of the points are predicted within an absolute error band of $20 \%$.
\end{abstract}

Key words:

Compressor, Expanders, Simulation, Leakage

\section{Introduction}

The simulation of positive-displacement compressors and expanders, here considered together as positive displacement machines, is of great importance from a research and development standpoint. With good simulation tools, it is possible to conduct optimization of the machine in order to improve the machine's performance and ultimately, the performance of the system into which it is integrated. This could be a heat pump or a power-generation cycle, such as an Organic Rankine Cycle.

One of the most challenging elements to model in positive-displacement compressors and expanders is leakage. The modeling of leakage flows is particularly difficult since in most cases the leakage gap widths are not known a priori, and the leakage flow is in general compressible, frictional, and the flow area changes over the length of the flow path. As a result, the use of simple models such as the isentropic nozzle model for leakage flow, results in large discrepancies when compared with more accurate detailed leakage flow modeling. More detailed models can capture all the physical effects simultaneously, but require a great deal of computational effort, precluding their implementation in comprehensive simulations.

In this paper, a hybrid method is proposed whereby the results from detailed leakage flow calculations are used to derive a simple correction term to the isentropic nozzle model. In this way, the computational efficiency of the

\footnotetext{
* Corresponding Author

Email addresses: ian.bell@ulg.ac.be (Ian H. Bell), groll@purdue.edu (Eckhard A. Groll), jbraun@purdue.edu (James E. Braun), wthorton@purdue.edu (W. Travis Horton)
}

isentropic nozzle model can be retained, but the accuracy of the detailed model can be introduced with only a slight loss in accuracy of prediction of the leakage flow rates under choked conditions.

The models are developed based on the scroll compressor geometry, but their formulation can be applied to any leakage flow calculation in positive displacement machines.

\section{Literature Review}

The typical baseline model for leakage in positive displacement machines is the compressible flow of a perfect gas through an isentropic converging nozzle. This model allows for choking when the Mach number reaches 1 at the throat and is typically used with empirical correction factors to compensate for static pressure losses in the flow path. The corrected isentropic flow model has been employed by a number of authors, including Margolis (1992), Puff (1992), Youn (2000), Lee (2002), Chen (2002), among many others. The primary motivating factor for the use of this model is its simplistic form since only one area, the throat area, is required. In addition the mass flow rate is explicitly obtained from the compressible mass flow expression, adding little computational overhead if implemented within a detailed simulation model. Typically this model is applied to both flank and radial leakage paths in scroll compressors. One of the challenges is determining the empirical discharge coefficient, and limited experimental data is available, but Cho et al. (2000) have investigated this problem and found a discharge coefficient of 0.1 fit their choked flow data well. This suggests that the isentropic flow model does not do a very good job of cap- 
turing the actual leakage mass flow rate since such a large correction is required.

One of the major shortcomings of the isentropic compressible flow model is that it does not take friction into account. However, in many positive displacement machines the leakage paths are relatively long compared to the leakage gap widths. In a typical scroll compressor, the lengths of the leakage flow paths can be up to 500 times as long as the gaps are wide. Therefore, friction can be expected to play a significant role in the leakage flow.

Frictional flow models can be categorized based on their treatment of compressibility; some models treat the fluid as incompressible, others as compressible. For incompressible flow, the pressure drop over the leakage path can be calculated from incompressible pipe flow relations, as suggested by Ishii (1996). Very good agreement is found with experimental measurements carried out on a specialized test stand built to test leakage characteristics. Yuan (1992) and Fan (1994) extended the incompressible flow with friction model to account for the inertial terms in the Navier-Stokes equations, which are neglected in the pipe flow analysis. However, they ended with an expression that needs to be numerically integrated over the flow path. They found that their model provides results that are superior to those of either isentropic compressible nozzle flow or to pipe flow. Kang (2002) found that using compressible adiabatic flow with friction (Fanno Flow) was a good match to the predictions of FLUENT results, and superior to the use of isentropic compressible nozzle flow. Suefuji (1992) also found good results by using Fanno flow through the leakage paths.

$\mathrm{Li}$ presented a model for the radial leakage flow based on the flow between cylinders (Li et al., 1992), but is missing units, while Yanagisawa (1985) presents the same model with the necessary description and units. Tseng (2006) also uses the same model.

Beyond the simplified models, there are a number of hybrid models that use elements from several models. Tojo (1986) used a combined converging isentropic nozzle/compressible frictional flow section to model the flow through the leakage paths. Afjei (1992) used superposition methods to calculate the volumetric flow through the leakage paths as a sum of the rolling, dragging, pressure driven, and flashing components.

The primary disadvantage of the detailed models is that while they can accurately predict the mass flow rate through the leakage gaps, they are computationally expensive. As a result, they cannot be practically employed in detailed compressor models. This is the motivation for the use of a frictional correction term to the isentropic nozzle model.

\section{Isentropic Nozzle Flow}

For the isentropic compressible nozzle flow model, the upstream and downstream pressures and the throat area are given and it is desired to calculate the mass flow rate. This model assumes that the fluid is an ideal gas with compressibility taken into account, but there is no friction. If the imposed pressure ratio is large enough to obtain sonic conditions at the throat of the nozzle, the flow is choked. The critical pressure ratio that yields sonic conditions at the throat of the nozzle is given by

$$
p_{r, c}=\left(1+\frac{k-1}{2}\right)^{k /(1-k)}
$$

where $k$ is the ratio of specific heats, given by $k=c_{p} / c_{v}$, evaluated at the upstream condition. The pressure ratio employed in the isentropic nozzle model is given by

$$
p_{r}=\left\{\begin{array}{cc}
p_{r, c} & p_{\text {down }} / p_{\text {up }} \leq p_{r, c} \\
p_{\text {down }} / p_{\text {up }} & p_{\text {down }} / p_{\text {up }}>p_{r, c}
\end{array}\right.
$$

Thus, the mass flow rate from the isentropic nozzle model is given by

$$
\dot{m}_{n}=A_{n} \sqrt{p_{u p} \rho_{u p}} \sqrt{\frac{2 k}{k-1}\left(p_{r}^{2 / k}-p_{r}^{(k+1) / k}\right)}
$$

where the parameter $A_{n}$, the nozzle throat area, is defined in the following sections for each flow path geometry.

\section{Detailed Model Development}

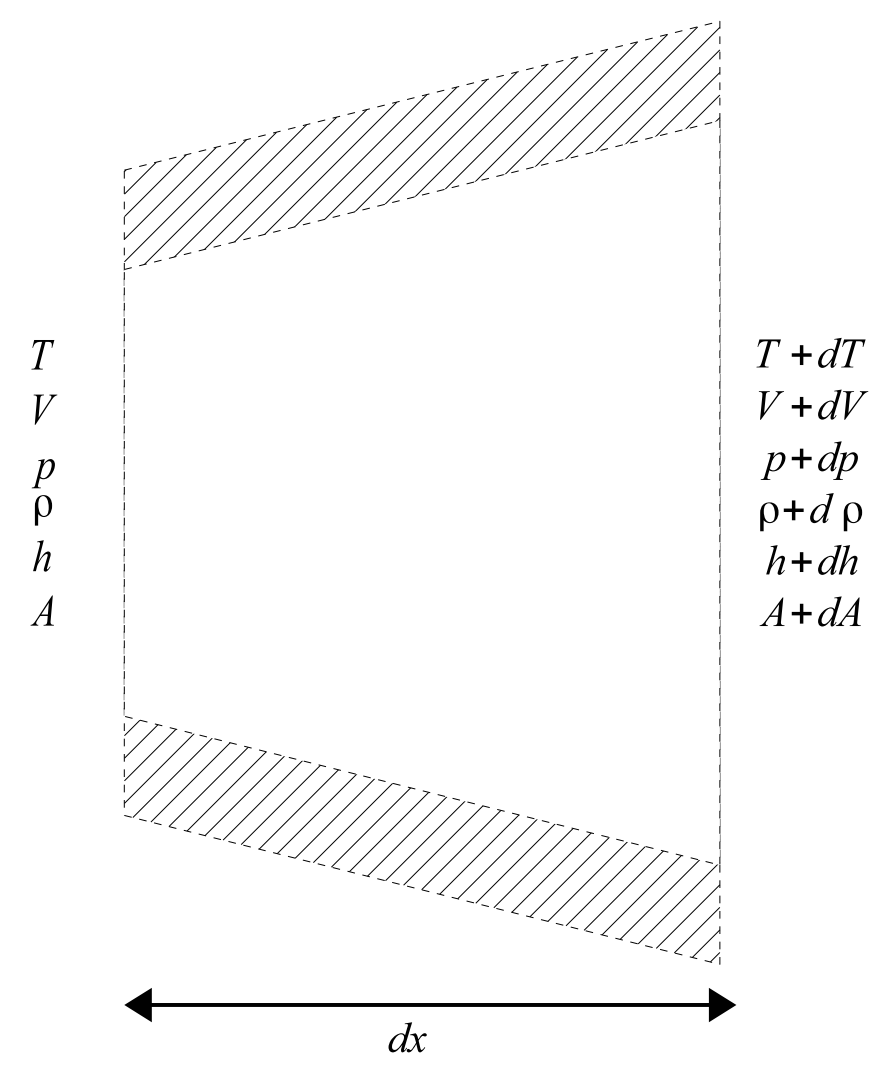

Figure 1: Schematic of differential element for 1-D detailed modeling 
In this section, the analysis required for the detailed model is developed. The analysis presented here largely follows that from the analysis of Wassgren (2009), but modifications are made for the addition of changing area and real gas properties. In the analysis that follows, the following assumptions have been employed:

- No heat transfer across the transverse boundary of the differential element

- No mass transfer across the transverse boundary of the differential element

- Variable area

- Compressible flow

- Real gas properties

- No oil, only gas

- One-dimensional flow

Figure 1 shows a schematic of the differential control volume of interest. The goal of the analysis in this section is to use conservation of mass, energy and momentum in order to derive a set of differential equations that govern the behavior of the gas in the control volume. This set of differential equations will then be integrated along the length of the control volume in order to calculate the pressure drop.

\subsection{Continuity}

The mass flow through the differential area can be given by

$$
\dot{m}=\rho V A
$$

and the mass flow rate $\dot{m}$ is constant since there is no mass addition or subtraction. Thus, taking the derivative of the mass flow rate with respect to the flow direction and setting it equal to zero yields

$$
\frac{1}{\rho} \frac{d \rho}{d x}+\frac{1}{V} \frac{d V}{d x}+\frac{1}{A} \frac{d A}{d x}=0
$$

\subsection{Momentum}

Conservation of momentum for the control volume is given by

$$
\int_{C S} \rho V^{2} d A=\sum F
$$

which yields

$$
\rho V \frac{d V}{d x}+\frac{d p}{d x}=-\frac{\rho V^{2}}{2} \frac{4 f_{F}}{D_{H}}
$$

when products of differentials are dropped. The Fanning friction factor is given by

$$
f_{F}= \begin{cases}\frac{24}{\operatorname{Re}_{\mathrm{d}}} & \operatorname{Re}_{\mathrm{d}}<1736.5 \\ \frac{\left(0.790 \ln \mathrm{Re}_{\mathrm{d}}-1.64\right)^{-2}}{4} & \mathrm{Re}_{\mathrm{d}}>1736.5\end{cases}
$$

which assumes a model for flow between infinite plates is reasonable. In the transitional Reynolds number regime, the turbulent and laminar Reynolds number curves are extrapolated to intersect at a Reynolds number of 1736.5 in order to ensure that the friction factor curve is monotonic, aiding numerical convergence. The Reynolds number is based on the local hydraulic diameter of the flow path, and is defined below for each flow path.

\subsection{Energy}

The differential element is treated as adiabatic with no transverse mass transfer, and as a result the stagnation enthalpy is constant. Thus, the conservation of energy is given by

$$
\dot{m}\left(h+\frac{V^{2}}{2}\right)-\dot{m}\left(h+d h+\frac{(V+d V)^{2}}{2}\right)=0
$$

which reduces to

$$
\frac{d h}{d x}+V \frac{d V}{d x}=0
$$

when the products of differentials are dropped.

\subsection{Thermodynamic Properties and Partial Derivatives}

In order to use the real gas properties of the refrigerant, the pressure and enthalpy need to be expanded in terms of $T$ and $\rho$. For a single-phase fluid, two state variables are needed to fix the state of the fluid, and the reference equations of state for most common fluids are expressed as a function of temperature and density. Thus, the partial derivatives of enthalpy and pressure can be explicitly obtained from the equation of state formulation. The differential of enthalpy is given by

$$
\begin{aligned}
& d h=\left(\frac{\partial h}{\partial T}\right)_{\rho} d T+\left(\frac{\partial h}{\partial \rho}\right)_{T} d \rho \\
& d p=\left(\frac{\partial p}{\partial T}\right)_{\rho} d T+\left(\frac{\partial p}{\partial \rho}\right)_{T} d \rho
\end{aligned}
$$

and dividing these equations by $d x$ yields

$$
\begin{aligned}
& \frac{d h}{d x}=\left(\frac{\partial h}{\partial T}\right)_{\rho} \frac{d T}{d x}+\left(\frac{\partial h}{\partial \rho}\right)_{T} \frac{d \rho}{d x} \\
& \frac{d p}{d x}=\left(\frac{\partial p}{\partial T}\right)_{\rho} \frac{d T}{d x}+\left(\frac{\partial p}{\partial \rho}\right)_{T} \frac{d \rho}{d x}
\end{aligned}
$$

The residual Helmholtz energy formulation of the properties gives the pressure and enthalpy in the forms

$$
\begin{gathered}
\frac{p}{\rho R T}=1+\delta\left(\frac{\partial \alpha^{r}}{\partial \delta}\right)_{\tau} \\
\frac{h}{R T}=\tau\left[\left(\frac{\partial \alpha^{0}}{\partial \tau}\right)_{\delta}+\left(\frac{\partial \alpha^{r}}{\partial \tau}\right)_{\delta}\right]+\delta\left(\frac{\partial \alpha^{r}}{\partial \delta}\right)_{\tau}+1
\end{gathered}
$$


where $\tau=T_{c} / T$ and $\delta=\rho / \rho_{c}$, and $T_{c}$ and $\rho_{c}$ are the critical temperature and density of the fluid, respectively. All of the fluid equations of state employed here (Span and Wagner, 1996; Span et al., 2000; Lemmon, 2003; TillnerRoth and Baehr, 1994) are of the residual Helmholtz energy formulation. Both the ideal gas Helmholtz energy contribution $\left(\alpha^{0}\right)$ and residual Helmholtz energy contribution $\left(\alpha^{r}\right)$ are in general functions of temperature and density. Lemmon (2000) gives the necessary partial derivatives of the pressure directly:

$$
\begin{gathered}
\left(\frac{\partial p}{\partial \rho}\right)_{T}=R T\left[1+2 \delta\left(\frac{\partial \alpha^{r}}{\partial \delta}\right)_{\tau}+\delta^{2}\left(\frac{\partial^{2} \alpha^{r}}{\partial \delta^{2}}\right)_{\tau}\right] \\
\left(\frac{\partial p}{\partial T}\right)_{\rho}=R \rho\left[1+\delta\left(\frac{\partial \alpha^{r}}{\partial \delta}\right)_{\tau}-\delta \tau\left(\frac{\partial^{2} \alpha^{r}}{\partial \delta \partial \tau}\right)\right]
\end{gathered}
$$

Calculations for partial derivatives of the enthalpy are given by the following terms

$$
\begin{gathered}
\left(\frac{\partial h}{\partial \rho}\right)_{T}=\frac{R T}{\rho_{c}}\left\{\begin{array}{c}
\tau\left[\left(\frac{\partial^{2} \alpha^{0}}{\partial \tau \partial \delta}\right)_{\delta}+\left(\frac{\partial^{2} \alpha^{r}}{\partial \tau \partial \delta}\right)_{\delta}\right] \\
+\left(\frac{\partial \alpha^{r}}{\partial \delta}\right)_{\tau}+\delta\left(\frac{\partial^{2} \alpha^{r}}{\partial \delta^{2}}\right)_{\tau}
\end{array}\right\} \\
\left(\frac{\partial h}{\partial T}\right)_{\rho}=\frac{1}{\rho}\left(\frac{\partial p}{\partial T}\right)_{\rho}-R \tau^{2}\left[\left(\frac{\partial^{2} \alpha^{0}}{\partial \tau^{2}}\right)_{\delta}+\left(\frac{\partial^{2} \alpha^{r}}{\partial \tau^{2}}\right)_{\delta}\right]
\end{gathered}
$$

\subsection{Solution for System of ODEs}

The set of equations to be solved are Equations 5, 7, 10, 13, and 14. The unknowns are the derivatives with respect to the flow direction $x$ of the variables $T, \rho, p, h$, and $V$.

With the use of a computer algebra system, the derivatives of each of the variables can be obtained and the solution for the system of differential equations is obtained as given in Table 1. Using this model, the flow area as a function of $x$ is needed to calculate the leakage flow rate in order to calculate the flow rate through a gap. With the flow area known, it is possible to numerically integrate the derivatives of Table 1 from the inlet of the flow path to the outlet of the flow path. The improved Euler method (also known as Heun's method or the Euler-Cauchy method) is used to integrate the system of equations along the flow path.

The problem for the calculation of the flow for a given leakage path is formulated with the upstream temperature, upstream pressure, and downstream pressure specified as known. The problem then is to find the mass flow rate that yields the downstream pressure at the outlet of the flow path. If the final pressure from the integration is equal to the downstream pressure, the mass flow rate has been appropriately calculated. If not, iteration is carried out using a secant solver to find the flow rate that yields the correct downstream pressure. The flow rate from the detailed model is constrained to be sub-sonic; that is, there are no normal shocks or choking in the flow path. The mass flow rate predicted by the detailed compressible flow model is given by $\dot{m}_{d}$.

For a given configuration, the ratio of the isentropic nozzle mass flow rate prediction to that of the detailed model is defined by

$$
M \equiv \frac{\dot{m}_{n}}{\dot{m}_{d}}
$$

\section{Leakage Path Geometry}

The analysis that follows in this section is primarily focused on the geometry of the scroll compressor as it has the most challenging geometric features to model. However, the resulting empirical correlations can be applied to other positive displacement machines with minimal modification.

\subsection{Radial Leakage}

Figure 2 shows the geometry of the simplified radial leakage path. The flow goes from a high pressure volume out to a lower pressure volume through an annulus with inner radius $r_{1}$ and outer radius $r_{2}$ with height $\delta$. In practice, the geometry of the scroll compressor is not exactly like that of the simplified geometry, but it is at least locally analogous. For application of the correlation in a detailed simulation model, average radii of curvature can be used in the place of $r_{1}$ and $r_{2}$.

The local flow area is equal to

$$
A=2 \pi \delta x
$$

where $x$ goes from $r_{1}$ to $r_{2}$. The derivative of $A$ is given by

$$
\frac{d A}{d x}=2 \pi \delta
$$

and the area used in the isentropic nozzle model is given by

$$
A_{n}=2 \pi \delta r_{1}
$$

The Reynolds number for the isentropic nozzle model correction is based on the upstream geometry and is given by

$$
\operatorname{Re}_{n}=\frac{\dot{m}_{n}}{\pi r_{1} \mu}
$$

where the viscosity $\mu$ is evaluated at the upstream state.

For the radial leakage, the characteristic length $L$ in the correlation is equal to $r_{2}-r_{1}$, which for a scroll compressor is simply equal to the scroll wrap thickness $t$.

In the detailed model, the local Reynolds number is needed. This Reynolds number is based on the hydraulic diameter which for the radial leakage path can be taken as $D_{H}=2 \delta$ since the transverse length is much greater than the gap width $\delta$. Thus, the local Reynolds number for the detailed model can be given by

$$
\operatorname{Re}_{d}=\frac{\dot{m}_{d}}{A} \frac{2 \delta}{\mu}
$$




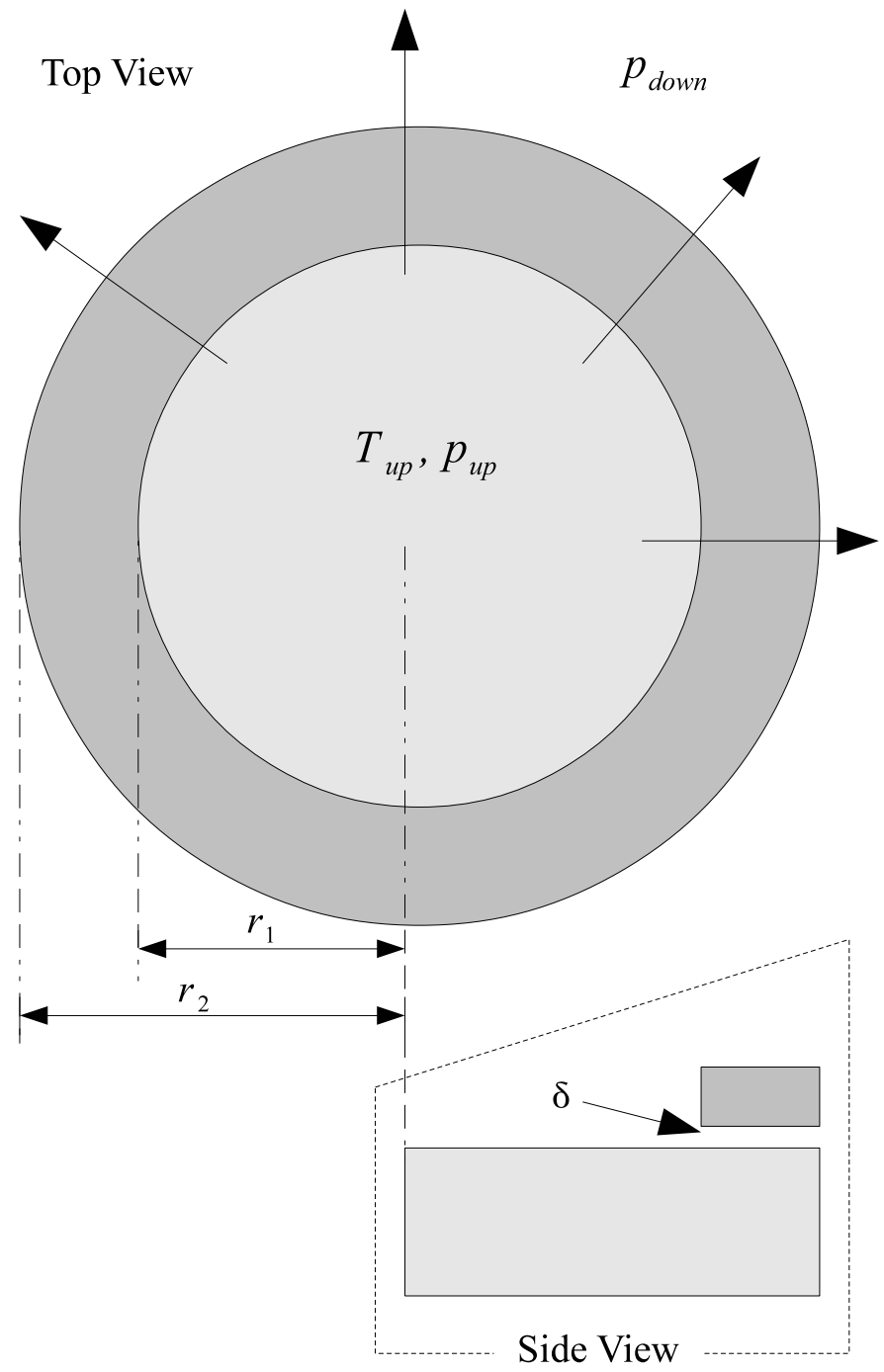

Figure 2: Radial leakage geometry schematic

where the mass flow rate $\dot{m}_{d}$ is a constant along the flow path by continuity and is iteratively calculated during the course of the solving process.

\subsection{Flank Leakage}

Figure 3 shows a schematic of a cross-section of the flow path considered for flank leakage. The flank leakage is flow through a converging-diverging nozzle formed by the conformal contact of the outer surface of one scroll wrap and the inner surface of the mating scroll wrap. The area of the flank flow path is given as a function of sweep angle $\phi$ (Yanagisawa and Shimizu, 1985) as

$A=h_{s}\left[R-(R-r-\delta) \cos \phi-\sqrt{r^{2}-(R-r-\delta)^{2} \sin ^{2} \phi}\right]$

where $\delta$ is the minimum gap width, and $r$ and $R$ are the radii of the inner and outer cylinders respectively. The $x$ coordinate corresponding to an angle $\phi$ is given by $x=\phi r$, the sweep angle for evaluation of the area can be obtained from $\phi=x / r$. Thus, the derivative of the area with respect to $x$ is

$$
\frac{d A}{d x}=\frac{h_{s}}{r}\left[(R-r-\delta) \sin \phi+\frac{(R-r-\delta)^{2} \sin \phi \cos \phi}{\sqrt{r^{2}-(R-r-\delta)^{2} \sin ^{2} \phi}}\right]
$$

For the flank leakage, the characteristic length $L$ is taken to be equal to $R-r$, which algebra shows is equal to the orbiting radius $r_{o}$ for the scroll compressor. The characteristic area for the isentropic nozzle model is equal to the minimum area of the flow path, or

$$
A_{n}=\delta h_{s}
$$

where $h_{s}$ is the height out of the page. The Reynolds number for the flank leakage flow is equal to

$$
\operatorname{Re}_{n}=\frac{2 \dot{m}_{n}}{h \mu}
$$

where the viscosity $\mu$ is evaluated at the upstream state.

Just as with the radial leakage, in the detailed model, the local Reynolds number is needed. In this case though, the Reynolds number varies over the length of the flow path. The hydraulic diameter is defined by

$$
D_{H}=\frac{4 A}{P}
$$

which can be reduced to

$$
D_{H}=\frac{2 A h}{h^{2}+A}
$$

The local Reynolds number for the detailed model is given by

$$
\operatorname{Re}_{d}=\frac{\dot{m}_{d}}{A} \frac{D_{H}}{\mu}
$$

where the mass flow rate $\dot{m}_{d}$ is a constant along the flow path by continuity and is iteratively calculated during the course of the solving process.

\section{Development of Correlation}

The detailed model and the isentropic nozzle model were evaluated for a range of conditions for a range of working fluids and both the flank and radial leakages. The same geometry was used for a range of fluids, detailed in Table 2. For each refrigerant, the high-side pressure was varied through the range shown, and then for each upstream pressure, the downstream pressure was decreased until a pressure ratio (given by $p_{r}=p_{u p} / p_{\text {down }}$ ) of $p_{r, \max }$ was reached. The gap width $\delta$ was varied in the range $5 \mu \mathrm{m}$ to $25 \mu \mathrm{m}$.

For the radial leakage, radii for $r_{1}$ in the range of 15.85 $\mathrm{mm}$ to $53.89 \mathrm{~mm}$ were investigated, and the length of the gap $(t)$ was varied from $2 \mathrm{~mm}$ to $10 \mathrm{~mm}$. 


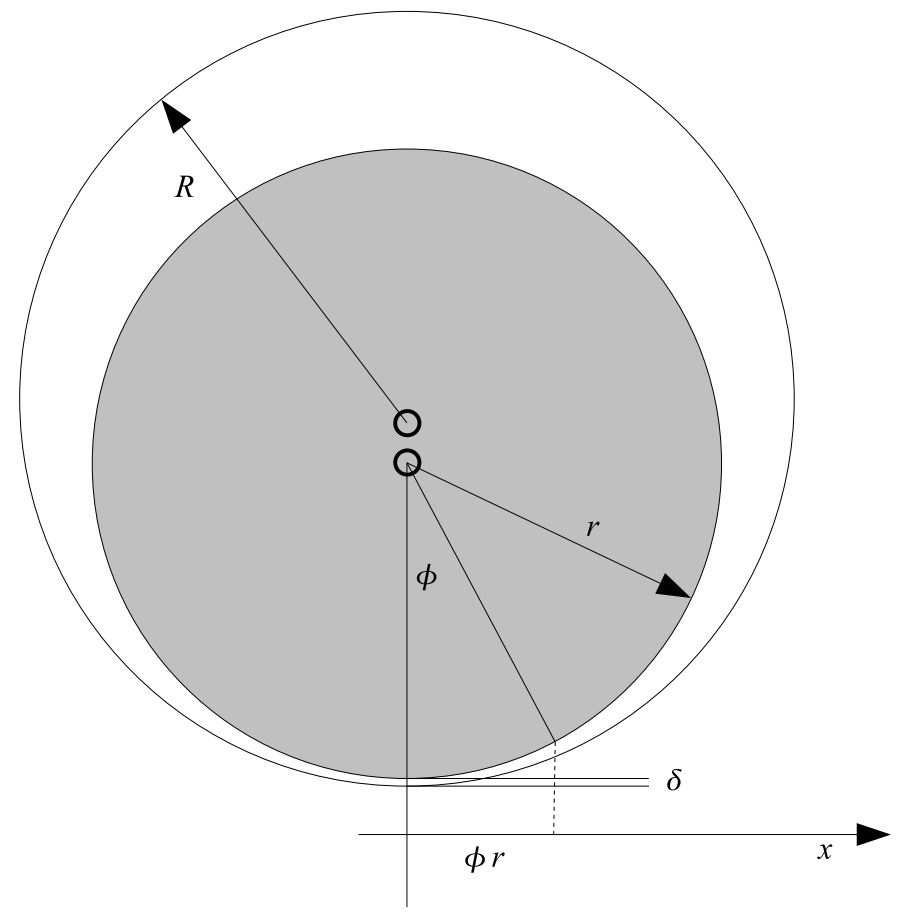

Figure 3: Flank leakage geometry schematic in cross-section

For the flank leakage, radii for $r_{1}$ in the range of 15.85 $\mathrm{mm}$ to $53.89 \mathrm{~mm}$ were investigated, and the length of the gap $(t)$ was varied from $2 \mathrm{~mm}$ to $10 \mathrm{~mm}$. The height of the gap $(h)$ was set to $32.89 \mathrm{~mm}$. The effects of the height are not important though as in general $h$ is much greater than $\delta$. In addition, for the flank leakage, the range of values for $x$ was taken to be from $-20 \mathrm{~mm}$ to $20 \mathrm{~mm}$. This range was selected to have the boundaries of the flow path sufficiently far away from the minimum area. It is assumed that the boundary layer remains attached in the expanding section of the flow path.

The results of these runs of the models form a database of two sets of data using the detailed and isentropic nozzle models. The ratio of the mass flow predictions is then the correction factor $M$ that is desired.

Table 2: Refrigerant states for development of frictional correction factor

\begin{tabular}{cccc}
\hline \hline Refrigerant & $p_{u p}[\mathrm{kPa}]$ & $p_{r, \max }[-]$ & $T_{u p}[\mathrm{~K}]$ \\
\hline Nitrogen & $1800-400$ & 1.5 & 320 \\
$\mathrm{CO}_{2}$ & $8000-6000$ & 1.5 & 320 \\
$\mathrm{R} 134 \mathrm{a}$ & $1500-400$ & 1.5 & 350 \\
$\mathrm{R} 410 \mathrm{~A}$ & $1500-1000$ & 1.5 & 350 \\
\hline
\end{tabular}

Figures 4 and 5 show the results of the dataset generated. Both the radial and flank flow paths exhibit similar behaviors. The larger $M$ is, the more important the frictional effects are. If $M$ is 100, the isentropic nozzle gives a prediction of the mass flow rate that is 100 times too high because the isentropic nozzle model neglects frictional effects. The frictional effects become more important at

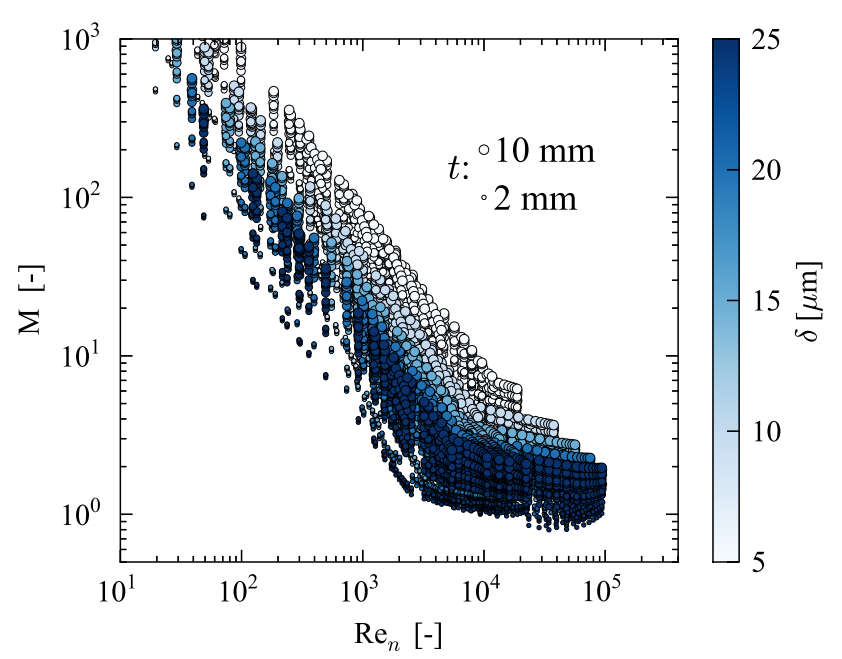

Figure 4: Ratio of isentropic nozzle model to detailed model for radial leakage as a function of Reynolds Number, gap width (color), and characteristic length (marker size)

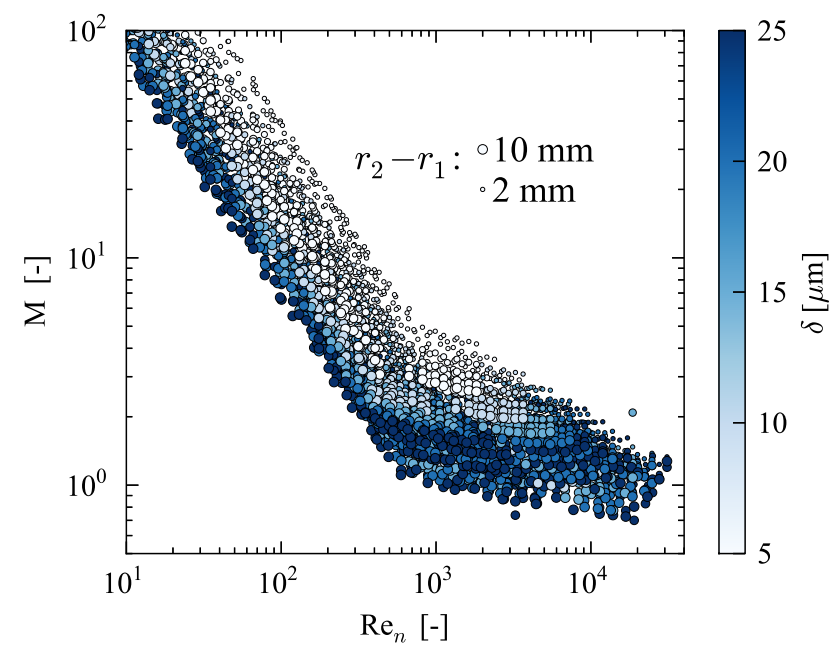

Figure 5: Ratio of isentropic nozzle model to detailed model for flank leakage as a function of Reynolds Number, gap width (color), and characteristic length (marker size)

lower Reynolds numbers.

For both flank and radial leakages, as the gap width goes down or the characteristic length increases, the frictional effects increase and the value of $\mathrm{M}$ increases.

\section{Developed Correlation}

A number of functional forms of the correlation were investigated, and the following form was obtained through regression and minimization of the root-mean-squared error of the correlation. The form of the correlation obtained is

$M=\frac{a_{0}\left(L^{*}\right)^{a_{1}}}{a_{2}\left(\delta^{*}\right)+a_{3}}\left[\xi\left(a_{4} \operatorname{Re}_{n}^{a_{5}}+a_{6}\right)+(1-\xi)\left(a_{7} \operatorname{Re}_{n}^{a_{8}}+a_{9}\right)\right]+a_{10}$ 
where the cross-over term $\xi$ is given by

$$
\xi=\frac{1}{1+\exp \left[-0.01\left(\operatorname{Re}-\operatorname{Re}^{*}\right)\right]}
$$

which is used to allow the curve fit optimizer to obtain two separate and continuous solutions for the low- and high-Reynolds numbers portions. The dimensionless characteristic length and dimensionless gap width are given by

$$
\begin{aligned}
L^{*} & =\frac{L}{L_{0}} \\
\delta^{*} & =\frac{\delta}{\delta_{0}}
\end{aligned}
$$

where the normalization parameters $L_{0}$ and $\delta_{0}$ are given by the values

$$
\begin{gathered}
\delta_{0}=10 \mu \mathrm{m} \\
L_{0}=0.005 \mathrm{~m}
\end{gathered}
$$

The same dimensionless parameters are used for both the flank and radial paths. The coefficients for the radial and flank flow paths are found in Tables 3 and 4 respectively. The other terms for the correlations are defined in the respective sections on leakage path geometry. The error of each correlation can be found in Figures 6 and 7. For the radial gap, the worst errors are found near the elbow between low- and high-Reynolds number and at high Reynolds numbers. For the flank gap, the worst errors are found at high Reynolds numbers. For the radial leakages, the average absolute error is $10.79 \%$ and the root-meansquared error in $M$ is 16.36 . For the flank leakage, the average absolute error is $14.54 \%$ and the root-mean-squared error in the prediction of $M$ is 2.25. For both correlations, at least $93 \%$ of the points are predicted within an absolute error band of $20 \%$.

Table 3: Coefficients for empirical correction term for radial leakage gap.

\begin{tabular}{llll}
\hline \hline & Value & & Value \\
\hline$a_{0}$ & $2.5932 \times 10^{4}$ & $a_{6}$ & $-1.2886 \times 10^{-2}$ \\
$a_{1}$ & $9.1483 \times 10^{-1}$ & $a_{7}$ & $-1.5120 \times 10^{2}$ \\
$a_{2}$ & $-1.7769 \times 10^{2}$ & $a_{8}$ & $-9.9967 \times 10^{-1}$ \\
$a_{3}$ & $-2.3705 \times 10^{-1}$ & $a_{9}$ & $1.6144 \times 10^{-2}$ \\
$a_{4}$ & $-1.7235 \times 10^{5}$ & $a_{10}$ & $8.2553 \times 10^{-1}$ \\
$a_{5}$ & $-1.2069 \times 10^{1}$ & Re* $^{*}$ & 5243.6 \\
\hline \hline
\end{tabular}

One of the motivating factors for the hybrid leakage calculation method is to embed the leakage model in a detailed compressor model in order to use the compressor model to predict its performance over a range of operating conditions. For that reason, the detailed model cannot be used directly due to the large amount of iteration needed and the commensurate increase in computational work required. For the flank leakages, using the hybrid method (isentropic nozzle with the correction term) is 1822 times
Table 4: Coefficients for empirical correction term for flank flow path.

\begin{tabular}{llcl}
\hline \hline & Value & & Value \\
\hline$a_{0}$ & -2.6397 & $a_{6}$ & $-5.1020 \times 10^{-1}$ \\
$a_{1}$ & $-5.6716 \times 10^{-1}$ & $a_{7}$ & $-1.2052 \times 10^{3}$ \\
$a_{2}$ & $8.3655 \times 10^{-1}$ & $a_{8}$ & -1.0294 \\
$a_{3}$ & $8.1057 \times 10^{-1}$ & $a_{9}$ & $6.8950 \times 10^{-1}$ \\
$a_{4}$ & $6.1740 \times 10^{3}$ & $a_{10}$ & 1.0961 \\
$a_{5}$ & -7.6091 & $\operatorname{Re}^{*}$ & 826.167178 \\
\hline \hline
\end{tabular}

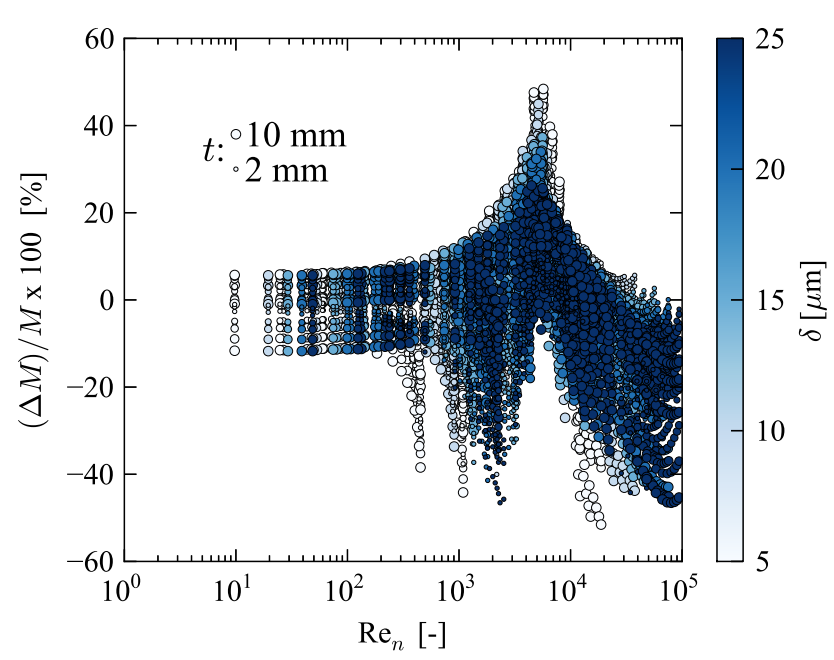

Figure 6: Error in radial correction term as a function of Reynolds Number, gap width (color), and characteristic length (marker size)

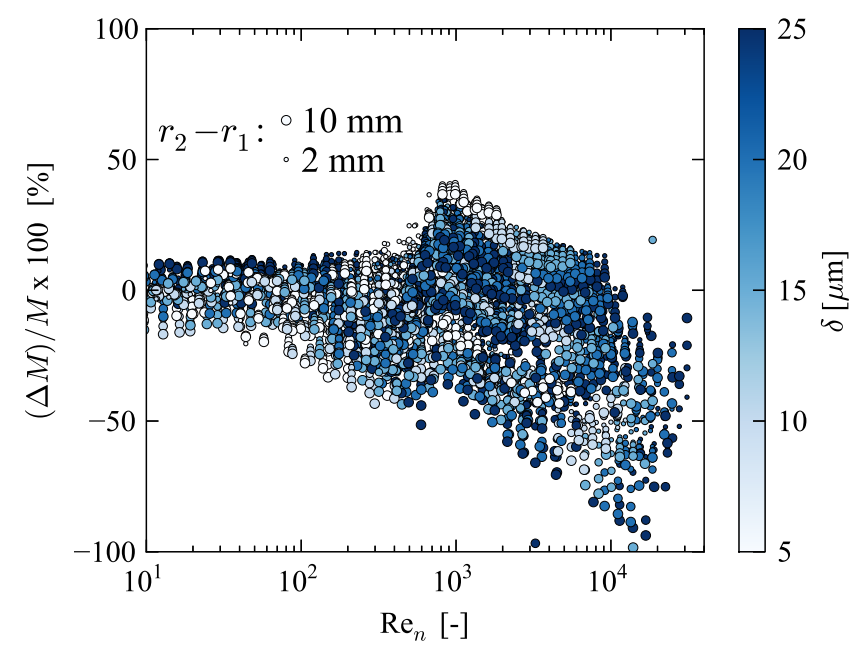

Figure 7: Error in flank correction term as a function of Reynolds Number, gap width (color), and characteristic length (marker size)

faster than using the detailed model. For the radial leakages, the hybrid method is 40 times faster than the detailed model.

Results for Nitrogen and $\mathrm{CO}_{2}$ flowing through a radial leakage gap are shown in Figures 8 and 9, respectively. The correction term works extremely well for $\mathrm{CO}_{2}$ since the isentropic nozzle model does not predict choking will 


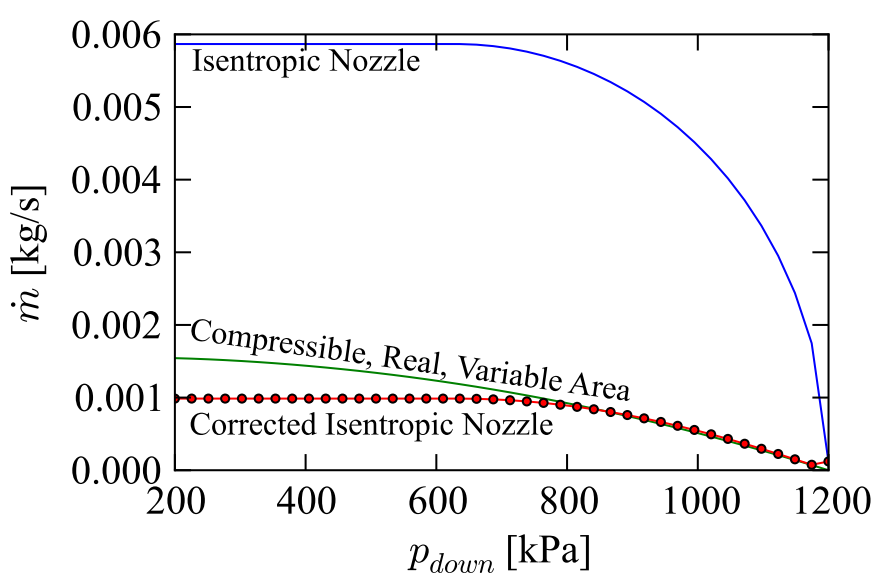

Figure 8: Nitrogen flow rates through the radial gap predicted by isentropic nozzle, detailed models, and corrected isentropic nozzle $\left(p_{u p}=1200 \mathrm{kPa}, T_{u p}=320 \mathrm{~K}, r_{1}=34.8 \mathrm{~mm}, t=4.66 \mathrm{~mm}, \delta=10\right.$ $\mu \mathrm{m})$

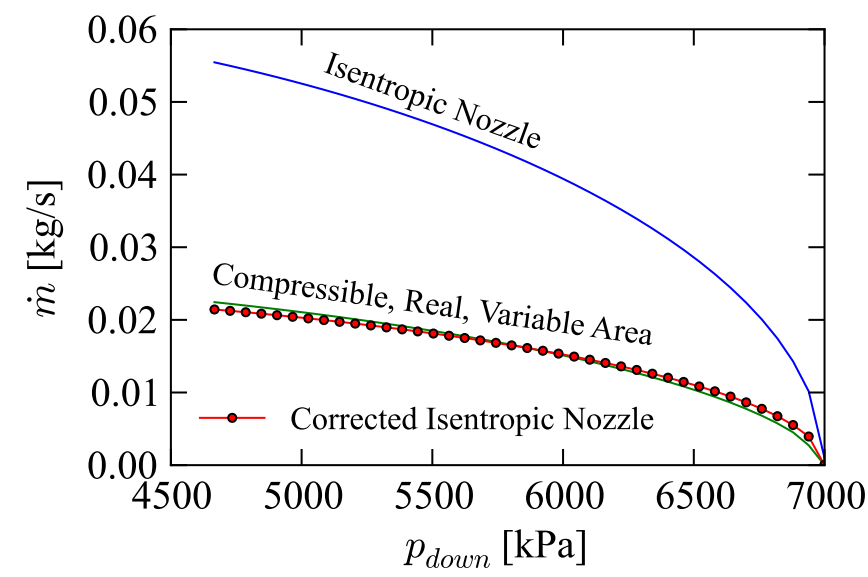

Figure 9: $\mathrm{CO}_{2}$ flow rates through the radial gap predicted by isentropic nozzle, detailed models, and corrected isentropic nozzle $\left(p_{u p}=7000 \mathrm{kPa}, T_{u p}=320 \mathrm{~K}, r_{1}=34.8 \mathrm{~mm}, t=4.66 \mathrm{~mm}, \delta=10\right.$ $\mu \mathrm{m})$

occur over the range of back pressures investigated. For nitrogen, the isentropic nozzle model predicts choking will occur, and for those points where choking does occur, the corrected model underpredicts the detailed model.

\section{Application to Other Compressor Geometry}

The correction terms presented here are most directly applicable to scroll compressors and expanders, but they can also be used in other types of positive displacement machines. For instance, the flank leakage correction term presented here is of the same form of the leakage around the rolling piston of rotary-type compressors. In any case, the above methodology can be used to derive a geometrically-appropriate leakage correction term for the compressor of interest.

\section{Conclusions}

A novel method has been proposed for the modeling of leakage in positive-displacement machines. This method is based on developing an empirical correction term to the isentropic nozzle model by using a large dataset of numerical results from detailed modeling of the leakage flow path. While derived primarily for scroll-type compressors and expanders, the correlations are general enough to be used for other types of positive-displacement machines. Additionally the correlations incur little computational overhead which makes them good candidates for use in a detailed positive-displacement simulation. Both correlations can predict greater than $93 \%$ of the points within $20 \%$.

\section{References}

Afjei, T., Suter, P., Favrat, D., 1992. Experimental analysis of an inverter-driven scroll compressor with liquid injection. In: 1992 Compressor Engineering Conference at Purdue University.

Chen, Y., Halm, N., Groll, E., Braun, J., 2002. Mathematical Modeling of Scroll Compressor. Part I- Compression Process Modeling. Int. J. Refrig. 25, 731-750.

Cho, N.-K., Youn, Y., Lee, B.-C., Min, M.-K., 2000. The Characteristics of Tangential Leakage in Scroll Compressors for Airconditioners. In: 15th International Compressor Engineering Conference at Purdue University. No. 807-814.

Fan, Z., Chen, Z., 1994. A calculating method for gas leakage in compressor. In: 1994 Compresor Conference at Purdue University.

Ishii, N., Bird, K., Sano, K., Oono, M., Iwamura, S., Otokura, T., 1996. Refrigerant Leakage Flow Evaluation for scroll compressors. In: 1996 Compressor Conference at Purdue University.

Kang, D. J., Kim, J. W., Sohn, C. B., 2002. Effects of leakage flow model on the thermodynamic performance of a scroll compressor. In: 2002 International Compressor Engineering Conference at Purdue University.

Lee, B.-C., Yanagisawa, T., Fukuta, M., Choi, S., 2002. A study on the leakage characteristics of tip seal mechanism in the scroll compressor. In: 2002 International Compressor Engineering Conference at Purdue University.

Lemmon, E., 2003. Pseudo-Pure Fluid Equations of State for the Refrigerant Blends R-410A, R-404A, R-507A, and R-407C. Int. J. Thermophys. 24 (4), 991-1006.

Lemmon, E., Jacobsen, R. T., Penoncello, S. G., Friend, D., 2000. Thermodynamic Properties of Air and Mixtures of Nitrogen, Argon, and Oxygen from 60 to $2000 \mathrm{~K}$ at Pressures to $2000 \mathrm{MPa}$. J. Phys. Chem. Ref. Data 29 (3), 331-385.

Li, H., Wang, D., Wang, H., Chen, P., 1992. Research of oil-injected scroll compressor working process. In: 1992 Compressor Engineering Conference at Purdue University. pp. 118b1-118b13.

Margolis, D. L., Craig, S., Nowakowski, G., Inada, M., 1992. Modeling and simulation of a scroll compressor using bond graphs. In: 1992 International Compressor Engineering Conference at Purdue University.

Puff, R., Krueger, M., 1992. Influence of the main constructive parameters of a scroll compressor on its efficiency. In: 1992 International Compressor Engineering Conference at Purdue University.

Span, R., Lemmon, E. W., Jacobsen, R. T., Wagner, W., Yokozeki, A., 2000. A Reference Equation of State for the Thermodynamic Properties of Nitrogen for Temperatures from 63.151 to $1000 \mathrm{~K}$ and Pressures to 2200 K. J. Phys. Chem. Ref. Data 29, 1361-1433.

Span, R., Wagner, W., 1996. A New Equation of State for Carbon Dioxide Covering the Fluid Region from the Triple-Point Temperature to $1100 \mathrm{~K}$ at Pressures up to $800 \mathrm{MPa}$. J. Phys. Chem. Ref. Data. 25, 1509-1596.

Suefuji, K., Shiibayashi, M., Tojo, K., 1992. Performance analysis of hermetic scroll compressors. In: 1992 International Compressor Engineering Conference at Purdue University. 
Tillner-Roth, R., Baehr, H. D., 1994. A International Standard Formulation for the Thermodynamic Properties of 1,1,1,2Tetrafluoroethane (HFC-134a) for Temperatures from $170 \mathrm{~K}$ to $455 \mathrm{~K}$ and Pressures up to $70 \mathrm{MPa}$. J. Phys. Chem. Ref. Data 23, $657-729$.

Tojo, K., Ikegawa, M., Maeda, N., Machida, S., Shiibayashi, M., Uchikawa, N., 1986. Computer modeling of scroll compressor with self adjusting back-pressure mechanism. In: 1986 International Compressor Engineering Conference at Purdue University.

Tseng, C.-H., Chang, Y.-C., 2006. Family design of scroll compressors with optimization. Appl. Therm. Eng. 26, 1074-1086.

Wassgren, C., 2009. ME 510 Gas Dynamics Class Notes (Purdue University). Boiler Copy Maker.

Yanagisawa, T., Shimizu, T., 1985. Leakage losses with a rolling piston type rotary compressor. II. Leakage losses through clearances on rolling piston faces. Int. J. of Refrig. 8 (3), $152-158$.

Youn, Y., Cho, N.-K., Lee, B.-C., Min, M.-K., 2000. The characteristics of tip leakage in scroll compressors for air conditioners. In: 2000 International Compressor Engineering Conference at Purdue University.

Yuan, X., Chen, Z., Fan, Z., 1992. Calculating model and experimental investigation of gas leakage. In: 1992 International Compressor Engineering Conference at Purdue University. 


\section{Nomenclature}

$a_{0}, a_{1}, \ldots \quad$ Correlation coefficients

$A \quad$ Area $\left(\mathrm{m}^{2}\right)$

$A_{n} \quad$ Nozzle throat area $\left(\mathrm{m}^{2}\right)$

$D_{H} \quad$ Hydraulic Diameter (m)

$f_{F} \quad$ Fanning friction factor (-)

$\mathrm{F} \quad$ Force (N)

$\mathrm{k} \quad$ Ratio of specific heats (-)

$L \quad$ Flow path length (m)

$L^{*} \quad$ Non-dimen. length (-)

$\mathrm{h} \quad$ Enthalpy $\left(\mathrm{J} \mathrm{kg}^{-1}\right)$

$\dot{m} \quad$ Mass flow rate $\left(\mathrm{kg} \mathrm{s}^{-1}\right)$

$\dot{m}_{d}$

$\dot{m}_{n}$

$\mathrm{M}$

$\mathrm{p}$

$\mathrm{P}$

$p_{\text {down }}$

$p_{u p}$

$p_{r}$

$p_{r, c}$

$r_{1}$

Pressure (Pa)
Mass flow rate from detailed model $\left(\mathrm{kg} \mathrm{s}^{-1}\right) \quad \delta$

Mass flow rate from isentropic nozzle model $\left(\mathrm{kg} \mathrm{s} \delta^{* 1}\right)$

Ratio of mass flow rates (-)

Perimeter (m)

Downstream pressure $(\mathrm{Pa})$

Upstream pressure $(\mathrm{Pa})$

Pressure ratio (-)

Critical pressure ratio (-)

Inner radius $(\mathrm{m})$

$\rho_{u p}$
Outer radius $(\mathrm{m})$

Small cylinder radius $(\mathrm{m})$

Big cylinder radius $(\mathrm{m})$

Specific gas constant $\left(\mathrm{J} \mathrm{kg}^{-1} \mathrm{~K}^{-1}\right)$

Reynolds Number (-)

Temperature (K)

Upstream temperature $(\mathrm{K})$

Position (m)

Velocity $\left(\mathrm{m} \mathrm{s}^{-1}\right)$

Ideal-gas Helmholtz energy (-)

Residual Helmholtz energy (-)

Gap width (m)

Reduced density (-)

Non-dimen. gap width (-)

Sweep angle (rad)

Upstream density $\left(\mathrm{kg} \mathrm{m}^{-3}\right)$

$\tau \quad$ Reduced temperature (-)

$\xi \quad$ Cross-over term (-)

Table 1: System of Equations developed

$$
\begin{aligned}
& \frac{d T}{d x}=-\Psi V^{2}\left[2 \rho A f_{F} V^{2}+\frac{d A}{d x}\left(\left(\frac{\partial p}{\partial \rho}\right)_{T} \rho D_{H}-\left(\frac{\partial h}{\partial \rho}\right)_{T} \rho^{2} D_{H}\right)-2\left(\frac{\partial h}{\partial \rho}\right)_{T} \rho^{2} A f_{F}\right] \\
& \frac{d p}{d x}=-\Psi\left[2 \rho A\left(\frac{\partial p}{\partial T}\right)_{\rho} f_{F} V^{4}+\rho^{2}\left(2 A f_{F}+\frac{d A}{d x} D_{H}\right)\left(\left(\frac{\partial p}{\partial \rho}\right)_{T}\left(\frac{\partial h}{\partial T}\right)_{\rho}-\left(\frac{\partial h}{\partial \rho}\right)_{T}\left(\frac{\partial p}{\partial T}\right)_{\rho}\right) V^{2}\right] \\
& \frac{d \rho}{d x}=-\Psi V^{2}\left[2 \rho^{2} A\left(\frac{\partial h}{\partial T}\right)_{\rho} f_{F}+\frac{d A}{d x}\left(\rho^{2} D_{H}\left(\frac{\partial h}{\partial T}\right)_{\rho}-\rho D_{H}\left(\frac{\partial p}{\partial T}\right)_{\rho}\right)\right] \\
& \frac{d h}{d x}=-\Psi\left[2 \rho A\left(\frac{\partial h}{\partial T}\right)_{\rho} f_{F} V^{4}+\rho \frac{d A}{d x} D_{H}\left(\left(\frac{\partial p}{\partial \rho}\right)_{T}\left(\frac{\partial h}{\partial T}\right)_{\rho}-\left(\frac{\partial h}{\partial \rho}\right)_{T}\left(\frac{\partial p}{\partial T}\right)_{\rho} V^{2}\right]\right. \\
& \frac{d V}{d x}=\Psi\left[2 \rho A\left(\frac{\partial h}{\partial T}\right)_{\rho} f_{F} V^{3}+\rho \frac{d A}{d x} D_{H}\left(\left(\frac{\partial p}{\partial \rho}\right)_{T}\left(\frac{\partial h}{\partial T}\right)_{\rho}-\left(\frac{\partial h}{\partial \rho}\right)_{T}\left(\frac{\partial p}{\partial T}\right)_{\rho}\right) V\right] \\
& \Psi=\left[A\left(\rho D_{H}\left(\frac{\partial h}{\partial T}\right)_{\rho}-D_{H}\left(\frac{\partial p}{\partial T}\right)_{\rho}\right) V^{2}+\rho A D_{H}\left(\left(\frac{\partial h}{\partial \rho}\right)_{T}\left(\frac{\partial p}{\partial T}\right)_{\rho}-\left(\frac{\partial p}{\partial \rho}\right)_{T}\left(\frac{\partial h}{\partial T}\right)_{\rho}\right)\right]^{-1}
\end{aligned}
$$

